\title{
A Rare Case Report of Hydronephrosis and Acute Kidney Injury Secondary to Gonadal Vein Thrombosis in a Young Male
}

\author{
Jason M. Sandberg, MD, Raymond B. Dyer, MD, and Majid Mirzazadeh, MD
}

\begin{abstract}
Background: Gonadal vein thrombosis (GVT) has been reported in association with malignancy and pelvic inflammatory conditions. Patients who develop GVT often require systemic anticoagulation to reduce the risk of pulmonary embolism and other local and distant thromboembolic effects. As the gonadal vein courses from the pelvis toward its outlet in the upper abdomen, its intimate relationship to the ureter in the setting of vascular pathology may pose a risk for urinary obstruction in the adult. We are reporting a rare case of GVT leading to ureteral obstruction and acute kidney injury (AKI) in a young otherwise healthy male and provide a review of similar literature.

Case Presentation: We describe a case of an otherwise healthy 29-year-old African American adult male presenting with acute diverticulitis and associated left GVT with no evidence of hypercoagulability, leading to ureteral obstruction, hydronephrosis, and AKI. Treatment with ureteral stent placement, endovascular intervention, and systemic anticoagulation led to resolution of his condition.

Conclusion: This report details a rare case of confirmed GVT in an adult male with resultant urinary obstruction. Decompression of the collecting system and treatment of the significant venous obstruction with surgical intervention, combined with medical systemic anticoagulation, were effective in reversing the underlying cause.
\end{abstract}

Keywords: endourology, urinary obstruction, hydronephrosis, gonadal vein thrombosis, acute kidney injury, radiology

\section{Introduction and Background}

G ONADAL VEIN THROMBOSIS (GVT) is a rare but welldescribed phenomenon associated with malignancy, including renal cell carcinoma and inflammatory bowel conditions. It occurs predominantly in postpartum women or in other obstetric and gynecologic conditions such as pelvic inflammatory disease, cesarean deliveries, and pelvic malignancies. Acute idiopathic GVT has also been reported in rare cases in the literature. Patients may be asymptomatic or present with abdominal pain, nausea, and malaise, although it has been historically difficult to differentiate the etiology of the symptoms from the concomitant underlying condition. Occlusion or thrombosis of the gonadal vein has classically been associated with varicocele in men, and typically presents on the left because of the acute angle of entry into the left renal vein. Right-sided varicocele often raises suspicion for retroperitoneal or renal malignancy, which may cause external compression of the gonadal vein as it joins the vena cava on this side.

Deep vein thrombosis (DVT) including that of the deep lower extremity veins, iliac veins, or the inferior vena cava (IVC) can be life threatening, if emboli migrate to the lungs. Prompt diagnosis and treatment is necessary to avoid serious complications and, when symptoms are present, provide relief. Rarely, other local effects may occur as well. We report a case of a previously healthy 29 -year-old man with acute thrombosis of his left common iliac and gonadal veins,

\footnotetext{
${ }^{1}$ Department of Urology, Wake Forest Baptist Health, Winston-Salem, North Carolina.

${ }^{2}$ Department of Radiology, Wake Forest University School of Medicine, Winston-Salem, North Carolina.
}

(C) Jason M. Sandberg et al. 2017; Published by Mary Ann Liebert, Inc. This is an Open Access article distributed under the terms of the Creative Commons Attribution License, which permits unrestricted use, distribution, and reproduction in any medium, provided the original work is properly cited. 
resulting in left hydroureter, hydronephrosis, and acute kidney injury (AKI).

\section{Case Presentation}

\section{Clinical history}

A healthy 29-year-old African American man presented to the emergency department (ED) with severe left lower quadrant abdominal pain and diarrhea. His only medical and surgical history included Achilles tendon rupture 4 months earlier, which required surgical repair. CT of the abdomen and pelvis showed distal descending colon diverticulitis, diminutive caliber IVC, and bilateral gonadal vein varices with acute GVT on the left side and associated hydroureteronephrosis (Fig. 1). Delayed excretion of contrast in the left renal unit was also observed. Laboratory tests were unremarkable except for increased creatinine up to $1.4 \mathrm{mg} / \mathrm{dL}$. There was no leukocytosis. Mesenteric duplex ultrasonography revealed superior mesenteric vein thrombosis. Coagulation studies did not reveal any abnormality. He was treated with antibiotics for diverticulitis and discharged home on warfarin. He presented back to the ED 1 week later with worsening abdominal pain, left flank pain, diarrhea, and nausea. Repeat CT scan showed resolving diverticulitis but persistent left GVT and persistent hydroureteronephrosis. International normalized ratio (INR) was 3.34 and creatinine rose to $1.71 \mathrm{mg} / \mathrm{dL}$. Magnetic resonance venography confirmed left gonadal vein occlusion with extension to the left common iliac vein with distal reconstitution of the left external and internal iliac veins (Fig. 2). The left renal vein remained patent. There was persistent left moderate hydronephrosis to the level of the distal ureter because of compression by the left gonadal vein in the pelvis.

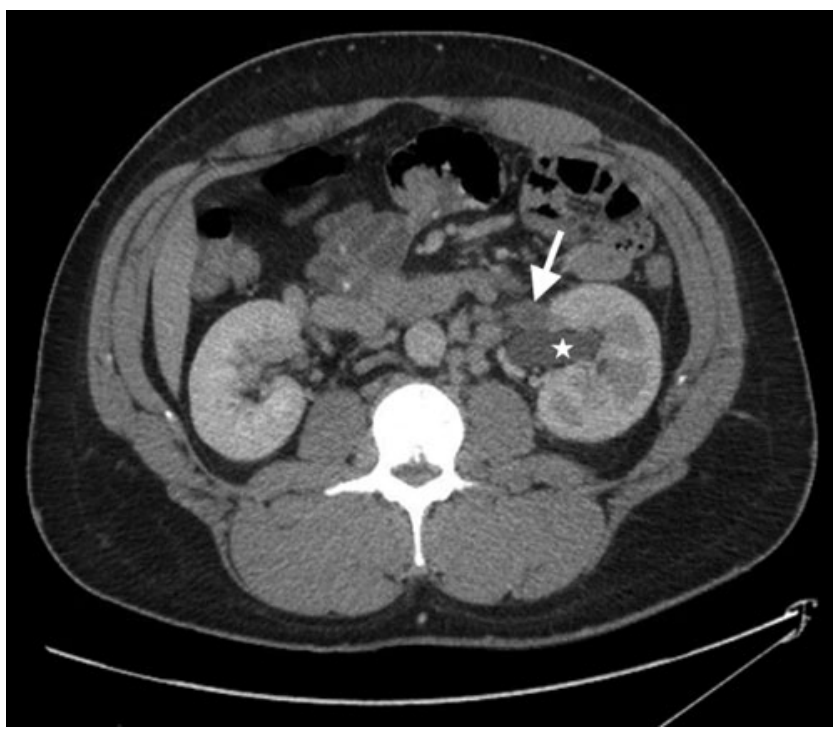

FIG. 1. Axial CT of the abdomen with contrast shows left hydronephrosis (star) and dilated left gonadal vein containing central thrombus (arrow) just proximal to the confluence with the left renal vein. Delayed left nephrogram progression indicates the presence of obstruction.

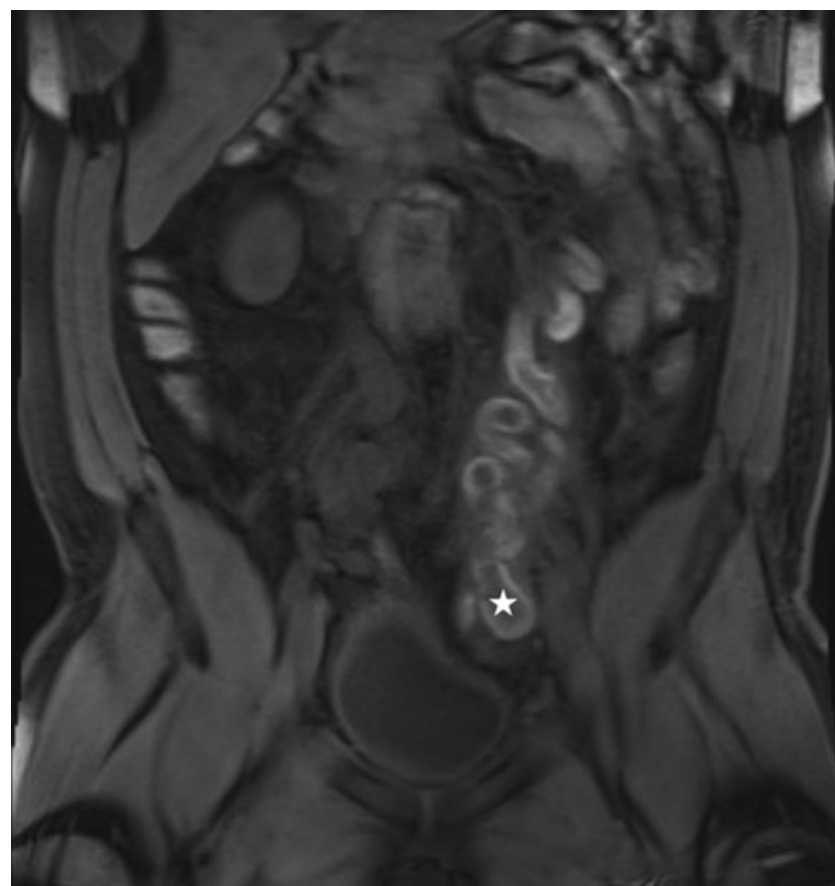

FIG. 2. Coronal T1-weighted contrast-enhanced magnetic resonance image of the abdomen and pelvis reveals acute left gonadal vein thrombosis (star).

Urology was consulted the following day. Physical examination was remarkable only for mild left costovertebral angle tenderness and mild left lower quadrant tenderness. His genitourinary examination was largely unremarkable without testicular tenderness or obvious varicocele. He was taken to the operating room for retrograde pyelogram, left ureteroscopy, and stent placement. There was clear narrowing of the distal left ureter with proximal lateral displacement, redundancy, and dilation (Fig. 3). It was impossible to pass the ureteroscope through the obstructed ureter safely. With stent placement, his creatinine decreased to $0.99 \mathrm{mg} / \mathrm{dL}$ within 72 hours. He was discharged on warfarin therapy but presented to the ED two more times over the next 3 weeks with bilateral lower extremity pain and was found to have left femoral DVT on duplex ultrasonography. His INR at this time was 1.83 and he was switched to rivaroxaban. He continued to have severe pain and was then taken to the operating room where he underwent stenting of his IVC, right common iliac vein, left common iliac vein, left external iliac vein, and thrombolysis with tissue plasminogen activator into the left common femoral artery by vascular surgery. He was returned to the operating room 48 hours later for further thrombectomy of the left iliofemoral system. Detailed hypercoagulable studies revealed slightly diminished antithrombin III concentration $(62 \%)$; he had a negative genetic thrombophilia panel including factor $\mathrm{V}$ Leiden and prothrombin gene mutation; negative Jak2 mutation, negative lupus inhibitor, normal homocysteine and lipoprotein (a), and a normal genetic panel.

He was discharged home on aspirin and rivaroxaban. Creatinine remained stable at $0.75 \mathrm{mg} / \mathrm{dL}$. CT 3 months later showed the indwelling ureteral stent and left common iliac vein stent with persistent bilateral varicose gonadal veins 


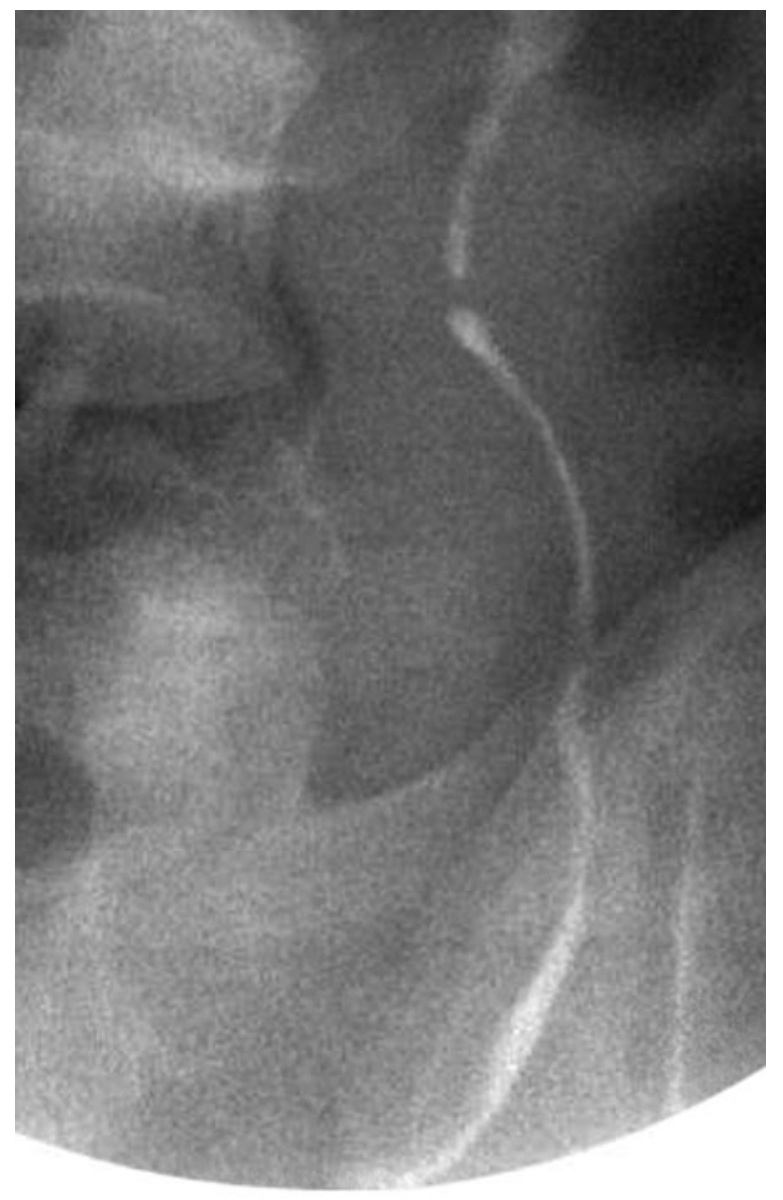

FIG. 3. Left retrograde pyelogram showing left distal ureteral narrowing with proximal hydroureter.

but also decreased inflammation, suggesting resolved thrombophlebitis (Fig. 4). He underwent stent exchange at that time, which was ultimately removed 6 months after initial presentation. At 7 months follow-up he was asymptomatic with no hydronephrosis on renal ultrasonography. He continues with planned life-long therapeutic anticoagulation, currently achieved with rivaroxaban.

\section{Discussion and Literature Review}

Previous literature has reported several cases of GVT secondary to acute gastrointestinal inflammatory (GI) conditions, but it is more commonly seen in the postpartum patient. Although simple varicosities of the gonadal vein have also been described, GVT does occur with a higher incidence in the oncology population, particularly in those with gastrointestinal/genitourinary cancers or metastatic disease. ${ }^{1}$

This is a rare case of ascending GVT causing hydronephrosis and AKI in an adult male. Alraies et al., recently detailed hydronephrosis in a male with an unprovoked DVT up to the level of the left common iliac vein, although no gonadal vein involvement was documented. ${ }^{2}$ A case of terminal ileitis associated with GVT in an adult male was also described by Schwartz and colleagues, but no ureteral obstruction was reported. ${ }^{3}$ Each of these patients presented with additional symptoms in-
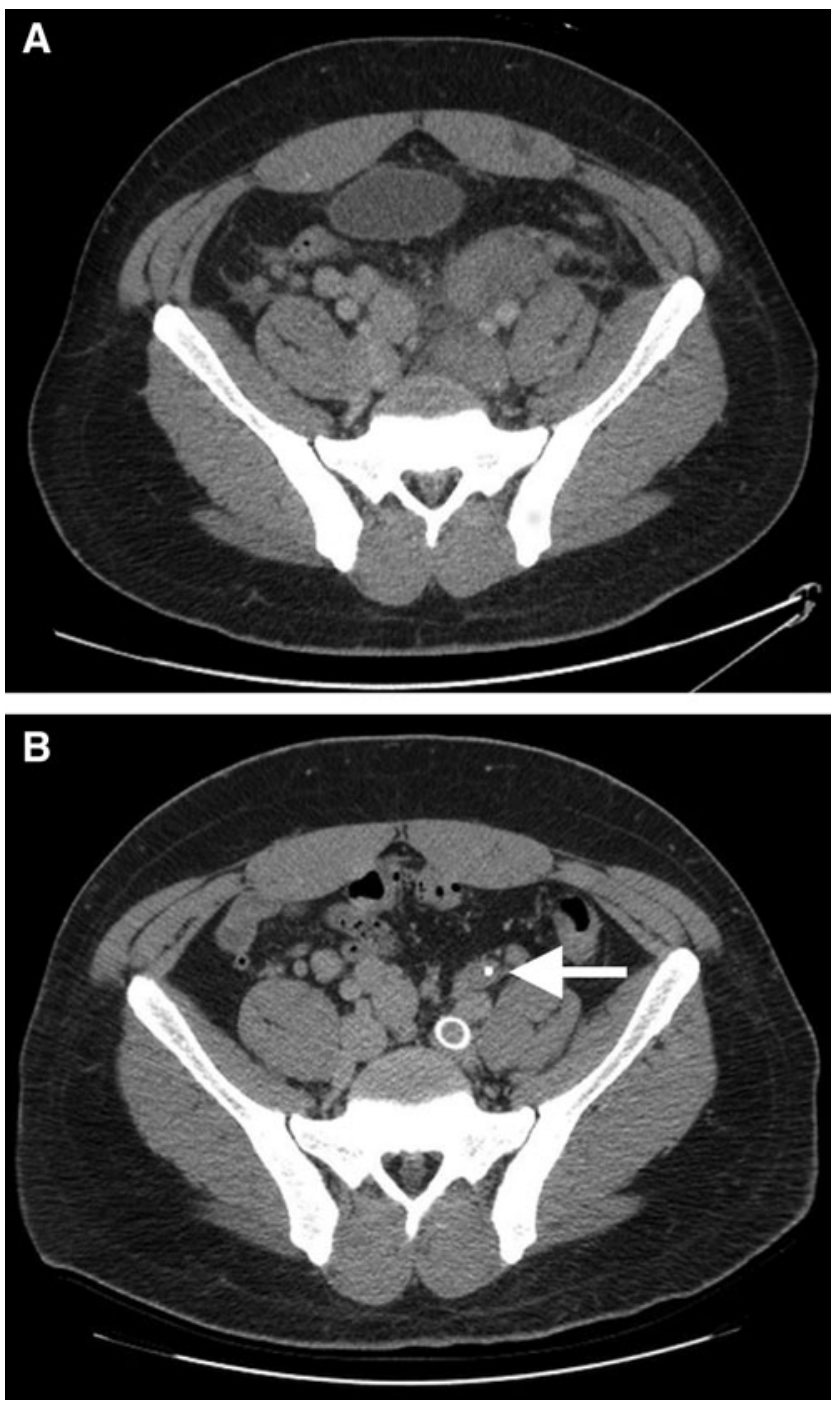

FIG. 4. Cross-sectional CT of the pelvis immediately cephalad to the branching of the internal and external iliac veins before (A) and after (B) left ureteral stent (arrow) and left iliac vein stent placements.

cluding lower extremity swelling, which was not present in our patient.

May-Thurner syndrome (MTS), although rare, has been well described and is caused by left iliac vein compression by the right iliac artery, resulting in ascending DVT. Nutcracker syndrome, in which there is symptomatic left renal vein compression between the aorta and superior mesenteric artery, is another circumstance that can lead to hematuria and/ or thrombosis of the renal and gonadal veins. ${ }^{4}$ Our case is similar to MTS in that our patient's thrombosis started distally and ascended proximally, although was not the result of a compressing iliac artery. Unique to this patient was the presence of ureteral obstruction, hydronephrosis, and AKI secondary to external compression from the thrombosed gonadal vein. In fact, his diminutive-caliber IVC was also postulated to have contributed to recruitment of collateral venous drainage and development of bilateral gonadal varicosities, which ultimately led to the thrombosis. 


\section{Conclusions}

This study illustrates a rarely reported case of confirmed GVT in an adult male with resultant urinary obstruction. Surgical intervention with placement of a ureteral stent and endovascular intervention proved sufficient to reverse the renal insult in the subacute setting, whereas outpatient therapy for the thrombus with oral systemic anticoagulation led to resolution of the thrombus and hydronephrosis after stent removal.

\section{Disclosure Statement}

No competing financial interests exist.

\section{References}

1. Tanasanvimon S, Garg N, Viswanathan C, et al. High prevalence of recurrent thrombosis in subsets of cancer patients with isolated gonadal vein thrombosis: A single center retrospective study. Thromb Res 2014;133:154-157.

2. Alraies MC, Kabach M, Shaheen K, Alraiyes AH. Unilateral leg swelling and hydronephrosis. QJM 2013;106:681-683.

3. Schwartz JH, Sclafani SJ, Glass TA, Sewell PE. Acute gonadal vein thrombosis secondary to terminal ileitis and thrombophilia. J Urol 2008;180:1124.

4. Weiner SN, Bernstein RG, Morehouse H, Golden RA. Hematuria secondary to left peripelvic and gonadal vein varices. Urology 1983;22:81-84.
Address correspondence to: Jason M. Sandberg, MD Department of Urology

Wake Forest Baptist Health

Medical Center Boulevard Winston-Salem, NC 27157-1094

E-mail:jmsandbe@wakehealth.edu

$\begin{aligned} & \text { Abbreviations Used } \\ \mathrm{AKI} & =\text { acute kidney injury } \\ \mathrm{CT} & =\text { computed tomography } \\ \mathrm{DVT} & =\text { deep vein thrombosis } \\ \mathrm{ED} & =\text { emergency department } \\ \mathrm{GI} & =\text { gastrointestinal inflammatory } \\ \mathrm{GVT} & =\text { gonadal vein thrombosis } \\ \mathrm{INR} & =\text { international normalized ratio } \\ \mathrm{IVC} & =\text { inferior vena cava } \\ \mathrm{MTS} & =\text { May-Thurner syndrome }\end{aligned}$

Cite this article as: Sandberg JM, Dyer RB, Mirzazadeh M (2017) A rare case report of hydronephrosis and acute kidney injury secondary to gonadal vein thrombosis in a young male, Journal of Endourology Case Reports 3:1, 119-122, DOI: 10.1089/cren.2017.0063. 